\title{
Observing the Structural Evolution in the Photodissociation of Diiodomethane with Femtosecond Solution X-Ray Scattering
}

\author{
Matthijs R. Panman $\odot,{ }^{1}$ Elisa Biasin $\odot,{ }^{2, \dagger}$ Oskar Berntsson $\odot,{ }^{1}$ Markus Hermann $\odot,{ }^{3}$ Stephan Niebling, ${ }^{1}$ Ashley J. Hughes, ${ }^{1}$ \\ Joachim Kübelø, ${ }_{1}^{1}$ Kalina Atkovska, ${ }^{3}$ Emil Gustavsson, ${ }^{1}$ Amke Nimmrich, ${ }_{1}^{1}$ Asmus O. Dohn, ${ }^{2}$ Mads Laursen, ${ }^{2}$ \\ Diana B. Zederkof, ${ }^{2}$ Alireza Honarfar $\odot,{ }^{4}$ Kensuke Tono, ${ }^{5}$ Tetsuo Katayama, ${ }^{5}$ Shigeki Owada, ${ }^{6}$ Tim B. van Driel, ${ }^{7}$ \\ Kasper Kjaer, ${ }^{7}$ Martin M. Nielsen, ${ }^{2}$ Jan Davidsson, ${ }^{8}$ Jens Uhlig $\odot,{ }^{4}$ Kristoffer Haldrup, ${ }^{2}$ \\ Jochen S. Hub, ${ }^{3}$ and Sebastian Westenhoff $\oplus^{1, *}$ \\ ${ }^{1}$ Department of Chemistry and Molecular Biology, University of Gothenburg, Box 462, 40530 Gothenburg, Sweden \\ ${ }^{2}$ Centre for Molecular Movies, Department of Physics, Technical University of Denmark, DK-2800 Lyngby, Denmark \\ ${ }^{3}$ Georg-August-Universität Göttingen, Institute for Microbiology and Genetics, Justus-von-Liebig-Weg 11, 37077 Göttingen, Germany \\ ${ }^{4}$ Department of Chemical Physics, Lund University, Box 124, S-2210, Lund, Sweden \\ ${ }_{5}^{5}$ Japan Synchrotron Radiation Research Institute, 1-1-1 Kouto, Sayo-cho, Sayo-gun, Hyogo 679-5198, Japan \\ ${ }^{6}$ RIKEN SPring-8 Center, 1-1-1 Kouto, Sayo-cho, Sayo-gun, Hyogo 679-5148, Japan \\ ${ }^{7}$ LCLS, SLAC National Laboratory, Menlo Park, California 94025, USA \\ ${ }^{8}$ Department of Chemistry, Ångström Laboratory, Uppsala University, Box 523, SE75120 Uppsala, Sweden
}

(Received 19 August 2020; revised 25 September 2020; accepted 19 October 2020; published 24 November 2020)

\begin{abstract}
Resolving the structural dynamics of the initial steps of chemical reactions is challenging. We report the femtosecond time-resolved wide-angle x-ray scattering of the photodissociation of diiodomethane in cyclohexane. The data reveal with structural detail how the molecule dissociates into radicals, how the radicals collide with the solvent, and how they form the photoisomer. We extract how translational and rotational kinetic energy is dispersed into the solvent. We also find that $85 \%$ of the primary radical pairs are confined to their original solvent cage and discuss how this influences the downstream recombination reactions.
\end{abstract}

DOI: 10.1103/PhysRevLett.125.226001

Understanding chemical reactivity requires resolving the reaction mechanism. Fundamental reaction events, such as the breaking, rearrangement, and formation of bonds as well as the interaction with solvent molecules, occur on femtosecond timescales. Femtosecond time-resolved spectroscopy has yielded a wealth of information about these steps. However, most femtosecond spectroscopic methods probe electronic states or vibrational modes, reporting only indirectly on molecular structure [1,2]. In particular, ubiquitous species in solution-state chemistry without covalent or ionic bonds, such as molecular encounter complexes, supramolecular assemblies, or solute-solvent complexes, are difficult to study [3]. Therefore, timeresolved techniques that are sensitive to atomic position are essential to further the understanding of chemical reactivity [4-13].

The photodissociation of diiodomethane $\left(\mathrm{CH}_{2} \mathrm{I}_{2}\right)$ is an ideal model reaction for studying elementary chemical

Published by the American Physical Society under the terms of the Creative Commons Attribution 4.0 International license. Further distribution of this work must maintain attribution to the author(s) and the published article's title, journal citation, and DOI. Funded by Bibsam. events. In apolar solvents, absorption of light at a wavelength of $266 \mathrm{~nm}$ results in impulsive $\mathrm{C}-\mathrm{I}$ bond scission, yielding $\left(\mathrm{CH}_{2} \mathrm{I}_{2} \rightarrow \mathrm{CH}_{2} \mathrm{I}^{\bullet}+\mathrm{I}^{*}\right)$ [14]. Within picoseconds, the fragments recombine to form a photoisomer $\left(\mathrm{CH}_{2} \mathrm{I}^{\bullet}+\mathrm{I}^{\bullet} \rightarrow \mathrm{CH}_{2} \mathrm{I}-\mathrm{I}\right)$ [15-17]. On nano- and microsecond timescales, the isomer and radical pair fragments undergo further bimolecular reactions to reform the parent molecule and $\mathrm{I}_{2}$ [15]. The photoisomer is the methylene transfer agent in the cyclopropanation of olefins with diiodomethane $[15,18]$.

Femtosecond optical spectra have shown that dissociation occurs impulsively within 200 fs $[19,20]$. However, the translational and rotational trajectories of the fragments cannot be retrieved from the optical spectra. The structure, solvent arrangement, and separation of the formed radical pairs remains unknown. Disagreement persists on whether the isomer is formed with a lifetime of $100 \mathrm{fs}$ [19], $1 \mathrm{ps}$ [20], or biphasically with 1 to 5 ps [15]. This is probably because the spectral signatures of the photoisomer and the vibrational relaxation of $\mathrm{CH}_{2} \mathrm{I}^{*}$ overlap. Nevertheless, the quantum yield of photoisomer formation has been determined to be between $\sim 70 \%$ and $95 \%$ for a variety of solvents at $100 \mathrm{ps}$ [15]. The remaining portion of the initial fragments are thought to be separated by solvent molecules so that they cannot recombine into the isomer. Solvent cage 
escape is a common explanation of incomplete reactions on fast timescales [20-23]. Even though this is a reasonable assumption, it is untested, as the structure of the radical pair cannot be probed with spectroscopic methods.

Femtosecond time-resolved WAXS.-To resolve the structural evolution of the fragments on femto- and picoseconds after photodissociation of $\mathrm{CH}_{2} \mathrm{I}_{2}$, we used time-resolved wide-angle $\mathrm{x}$-ray scattering (TRWAXS) at an $\mathrm{X}$-ray free electron laser (XFEL). We recorded the data at the Linear Coherent Light Source (LCLS) and Spring-8 Angstrom Compact free electron LAser (SACLA). $\mathrm{CH}_{2} \mathrm{I}_{2}$ in cyclohexane $\left(50 \mathrm{mmol} \mathrm{L}^{-1}\right)$ was supplied to the experiment in a liquid jet (see Supplemental Material and Fig. S1 [24] for details of the sample preparation and delivery). The reaction was triggered with femtosecond optical laser pulses (266 nm, <60 fs FWHM duration), which overlapped with the x-ray pulses $(9.5 \mathrm{keV}$ photon energy, < 50 fs FWHM duration) in the sample jet. The optical excitation density was $32 \mathrm{~mJ} \mathrm{~cm}^{-2}$, comparable to the $20 \mathrm{~mJ} \mathrm{~cm}^{-2}$ used in past ultrafast optical studies [15] and in the linear excitation regime of the sample (see Supplemental Material Fig. S2 [24]). The x-ray scattering was recorded at defined delay times relative to the laser pulse with an effective time resolution of 90 fs using the timing diagnostic at the beam line [60]. The signal processing of the diffuse 2D scattering images is described in the Supplemental Material [24]. The TRWAXS signal, due to solvent heating, was subtracted from the difference data prior to fitting the difference scattering (see Supplemental Material and Figs. S5-S7 [24]). In the following, we present the data recorded at the LCLS, but the data obtained at SACLA confirms the conclusions drawn (see Supplemental Material Figs. S13, S14, and S16 [24]).

Direct visualization of a dissociating molecule.-The azimuthally integrated difference scattering curves [Fig 1(c)] arise predominantly from the evolution of the distance of the two electron-rich I atoms. The observed momentum transfers $(q)$ range from 0.4 to $4.8 \AA^{-1}$, which is sufficient for a high-quality structural determination $[61,62]$. Figure $1(d)$ shows the data in real space, which allows inspection of the evolution of the interatomic distances in a model-free fashion [63]. The $r^{2} \Delta S(r, t)$ signal shows a negative feature with a time-dependent minimum between 3 and $4 \AA$, which is due to the depletion of the $\mathrm{I} \cdots \mathrm{I}$ distance of the $\mathrm{CH}_{2} \mathrm{I}_{2}$ ground state. The position of this negative peak ought to be time independent, and the observed shift is due to the overlapping positive signals. A positive difference-scattering feature appears at $100 \mathrm{fs}$ and shifts to large distances within a few hundred femtoseconds (orange band), which we assign to the impulsive (ballistic) separation of the I atom from the parent molecule. Impulsive separation is expected from optical spectra and the timescale is reasonable for the expected time of flight for an atom to hit the neighboring

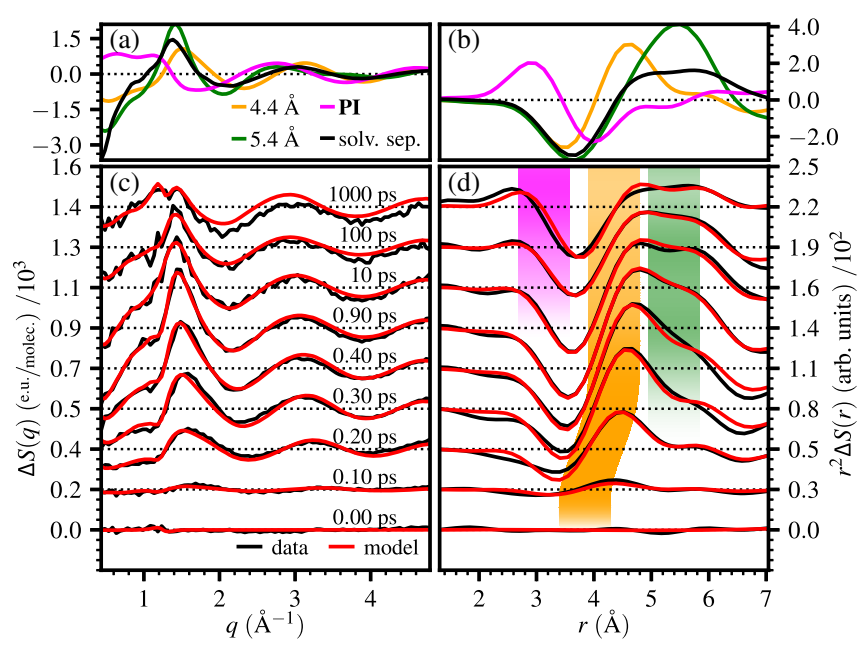

FIG. 1. (a), (b) The difference scattering profiles of refined I $\cdots$ I distances in reciprocal $(q)$ and real-space $(r)$. (c), (d) The difference scattering $[\Delta S(q)$, black] and difference scattering obtained from the structural refinement (red) are shown for selected time delays in reciprocal and real space. The conversion between reciprocal and real space was achieved by sine-Fourier transformation. The colored bands follow the refined I $\cdots I$ distances and are explained in the text.

molecule $[15,20]$. A pronounced positive shoulder arises after $\sim 1$ ps (green band) in between 5 and $6 \AA$. Both positive peaks correspond to photoinduced structures within the first solvent shell, which has a diameter of approximately $7 \AA$, as estimated from our molecular dynamics simulations (see Supplemental Material [24] for details). We assign the two distances to $\mathrm{I}^{\bullet}$ positioned at the I- and $\mathrm{CH}_{2}$-hemisphere of the $\mathrm{CH}_{2}{ }^{*}$ fragment due to the rotation of the $\mathrm{CH}_{2} \mathrm{I}^{\circ}$ fragment. A third positive feature is visible at shorter I … I distances compared to the ground state bleach (magenta band). The signal overlaps with the ground state bleach and is first visible as a positive indent of the negative band from around $\sim 400 \mathrm{fs}$ and more pronounced at later times. This marks the formation of the photoisomer (PI) $[16,64,65]$. Features associated with the rearrangement of the solvent are found at $r \geq 6.5 \AA$.

Structural refinement.-We continued our analysis by refining iodine distances (I $\cdots \mathrm{I})$ and their time-dependent concentrations $[A(t)]$ against the difference scattering in reciprocal space. The fits were obtained by selection from a model library of 124 theoretical scattering curves for two iodine atoms constrained at distances spanning 0 to $15 \AA$ (see Supplemental Material Fig. S17 [24]). The theoretical scattering was computed as $\Delta S=\sum_{i} A(t)_{i} \cdot\left[S_{i}(\mathrm{I} \cdots \mathrm{I})-S_{g s}(\mathrm{I} \cdots \mathrm{I})\right]$, where three contributions are considered for the calculation of $S(\mathrm{I} \cdots \mathrm{I})$ : the solute, solvent-solute, and solvent scattering terms. The solute term was computed using the Debye equation for two iodine atoms. Following a common approach $[16,66]$, the solvent-solute term was extracted from molecular dynamics trajectories of iodine atoms in cyclohexane. 
The solvent-solvent term was also extracted from the same molecular dynamics trajectories (see Supplemental Material Eqs. S6, S7, and S10 [24]). The scattering of ground state was assumed to be independent of time.

Following our qualitative assignments, we included four I … I distances, corresponding to three photoinduced species in the structural refinement. One distance represented the photoisomer and another one a radical pair, which was separated by solvent molecules. We fixed the latter distance to $100 \AA$, since the difference scattering in our detected range is invariant for I ... I separation $\gtrsim 15 \AA$. The remaining two I $\cdots$ I distances represent the geminate radical pair within the same solvent shell. The $\mathrm{I}^{*}$ is most likely distributed continuously around the $\mathrm{CH}_{2} \mathrm{I}^{\circ}$, but using the two dominating distances, which describe the $\mathrm{I}^{*}$ fragment on either side of the $\mathrm{CH}_{2} \mathrm{I}^{\bullet}$ hemisphere, is a practical approximation. We obtained optimal fits for I … I distances of the geminate pair at $4.35 \pm 0.03 \AA$ and $5.40 \pm 0.02 \AA$, of the ground state at $3.62 \pm 0.01 \AA$, and for the isomer at $3.13 \pm 0.04 \AA$ [red curves in Figs. 1(c) and 1(d), see Figs. 1(a) and 1(b) and Supplemental Material Fig. S10 [24] for the individual scattering contributions of each species]. The isomer and ground state distances agree with previous synchrotron studies in methanol $[16,65]$ and cyclohexane [64]. There are no significant discrepancies between the fit and data, indicating that the minimal model is sufficient to reproduce the experiment.

Kinetic analysis at $t<1 \mathrm{ps}$. - In order to describe the initial dissociation of the molecule, the short I . . I distance of the geminate pair was allowed to increase linearly for $t<500$ fs. Figure 2(a) shows that the fragments separate by $0.7 \pm 0.1 \AA$ over a period of $300 \mathrm{fs}$ before stopping at the static I … I separation of $4.35 \pm 0.03 \AA$. The instrument response function (IRF) as extracted from the anisotropic scattering (see Supplemental Material [24] for details) was shorter than the observed flight of the fragments [Fig. 2(b)]. Thus, the observation reflects how the $\mathrm{I} \cdots \mathrm{I}$ bond in $\mathrm{CH}_{2} \mathrm{I}_{2}$ breaks; how the fragments continue their flight; and how they finally collide with the solvent

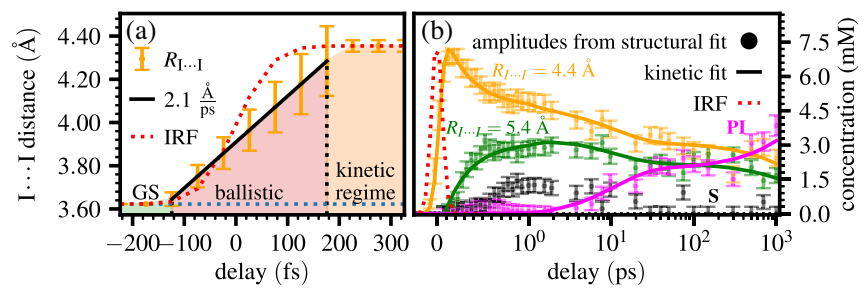

FIG. 2. (a) the I … I separation of the geminate pair species as a function of time $t$. The colored areas separated by dashed lines denote different dynamic regimes. The solid black line is a guide to the eye. The red-dotted line represents the instrument response function (see Supplemental Material Fig. S9 [24]) [8]. (b) time dependence of the amplitudes obtained by the structural refinement (Fig. 1, dots) and the least-squares fit of the kinetic model (lines). shell. The fragments travel with a relative velocity of $2.1 \pm 0.4 \AA \mathrm{As}^{-1}$ corresponding to $210 \pm 40 \mathrm{~ms}^{-1}$.

Next, we inspect the time-dependent concentrations obtained by the structural refinement [Fig. 2(b)]. We observe that the short I … I distance of the geminate pair rises, limited by the instrument response function (yellow markers). The concentration of the longer distance of the geminate pair rises concomitantly with the decay of the short distance (green markers). This supports our interpretation that the longer distance of the geminate pair is produced by rotation of the $\mathrm{CH}_{2} \mathrm{I}^{*}$ fragment. The rotation of this fragment increases the distance between the two iodine atoms because the $\mathrm{CH}_{2}$ moiety of the $\mathrm{CH}_{2} \mathrm{I}^{\circ}$ takes up space between them. A high-level quantum mechanics calculation shows that the distances are reasonable [see Supplemental Material Figs. S11 (a) and (b) [24]]. Figure 2(b) also reveals a delayed rise of the distance corresponding to the solvent-separated radical pair (black), which reflects the fragments having to travel a longer distance to escape the solvent cage. The relative amplitude of this species is a minor component of the total product species, indicating that the majority of the radical pairs stop at the first solvent shell. The distance corresponding to the photoisomer does not appear within the first picosecond (magenta; see below).

We cast these observations into a comprehensive kinetic model (Eqs. S1-S30, and see Supplemental Material Fig. S15 [24] for a visual representation) and performed a least-squares fit to the time-dependent amplitudes [solid lines, Fig. 2(b)]. We found that the rotational transition from the short to the long distance in the geminate pair occurs with a lifetime of $\tau_{r}=0.77 \pm 0.07 \mathrm{ps}$. This $\tau_{r}$ corresponds to a rotational lifetime of $0.25 \pm$ $0.02 \mathrm{ps} \mathrm{rad}^{-1}$ and an angular velocity of $4.1 \mathrm{ps}^{-1}$, which is many times faster than the predicted rotationalcorrelation lifetime of the steady-state $\mathrm{CH}_{2} \mathrm{I}^{\bullet}$ fragment as estimated from the Stokes-Einstein-Debye equation (16 $\mathrm{ps} \mathrm{rad}^{-1}$, see Supplemental Material Eqs. S31-S34 [24]). Therefore, the rotational motion is photoinduced and not caused by the equilibrium motion of the radicals. Interestingly, $\tau_{r}$ is longer than the collision time with the solvent $(0.34 \pm 0.07 \mathrm{ps})$, which implies that the rotational energy is not fully dissipated during the initial collision with the solvent shell. This illustrates that the geometry of the solute-solvent interaction is important for determining the dissipation of energy into the solvent.

The partitioning of excess energy.-Photoexcitation of a molecule transfers it into a high-lying electronic state, where the excess energy is partitioned into translational, rotational, and vibrational degrees of freedom, before energy dissipates to the solvent. The separation velocity $\left(2.1 \pm 0.4 \mathrm{ps}^{-1}\right)$ gives a translational kinetic energy of $0.02 \mathrm{eV}$ (see Supplemental Material Eqs. S35-S36 [24]). This represents $\sim 0.5 \%$ of the $4.66 \mathrm{eV}$ excitation photon energy or $\sim 7 \%$ of the $0.28 \mathrm{eV}$ kinetic energy found in the 
gas-phase dissociation reaction [67]. The rotational kinetic energy of the $\mathrm{CH}_{2} \mathrm{I}^{\bullet}$ fragment was computed from the angular velocity $\left(4.1 \mathrm{ps}^{-1}\right)$ and is $0.55 \mathrm{eV}$, representing $\sim 12 \%$ of the $4.66 \mathrm{eV}$ excitation photon energy (see Supplemental Material Eqs. S37-S39 [24]). Thus, the translational energy partition is smaller than the rotational by a factor of 24. In part, this may be due to the choice of photoexcitation energy. It may also be that our measurement rather underestimates the separation velocity, because it is close to the time resolution of the experiment. However, it is consistent that we find that a low amount of solvent separated species as the initial translational energy of the fragments is insufficient to break through the solvent barrier. These considerations also show that the notable portion of the excess excitation energy is held as vibrational energy.

Kinetic analysis at $t>1 \mathrm{ps}$.-Now, inspecting the kinetics at $(t>1 \mathrm{ps})$, we observe that the two I $\cdots$ I distances of the geminate pair decay biphasically and concurrently [Fig. 2(b)]. The characteristic I …I distance of the photoisomer is formed concomitantly with the biphasic decay of the geminate pair $\left(\tau_{1}=8 \pm 1\right.$ ps and $\tau_{2}=2.8 \pm 0.5 \mathrm{~ns}$ ). This is slower than what was concluded from optical spectroscopy $[15,19,20]$. We note that optical spectroscopic studies have never reached agreement on the timescale of isomer formation. We consider the results obtained from the TRWAXS experiment to be reliable given that the peaks of the photoisomer are well separated from other peaks in the TRWAXS data [Fig. 2(b)]. The kinetics of heat generation in the sample also corroborates the biphasic formation of the photoisomer (see Supplemental Material and Fig. S8 [24]).

Observation of a long-lived, but chemically inactive, geminate radical pair.-Surprisingly, we observe that the geminate pair is present for hundreds of picoseconds [Figs. 2(b) and 1(b)]. The concentration of the solventseparated pair never exceeds $15 \%$ of the total product species. We also observe a decrease of the solventseparated species after $t>10 \mathrm{ps}$, but this might be due to the decrease in data statistics after this time point [68]. We tested the robustness of the fits by selectively removing species from the model for delays $t>50 \mathrm{ps}$. We found that, when removing each of the geminate pair distances, the goodness of the fit $\left(\chi^{2}\right)$ was notably reduced, but when the solvent separated species was excluded, only a very minor reduction in the goodness was observed (see Supplemental Material Fig. S18 [24]).

Strong dispersion forces confine the radical pairs within the same solvent shell.-The existence of geminate pairs for hundreds of picoseconds indicates that an attractive force holds the radicals together. We investigated this interaction between $\mathrm{CH}_{2} \mathrm{I}^{*}$ and $\mathrm{I}^{\bullet}$ fragments with quantum chemical calculations at the complete active space selfconsistent field multireference configuration interaction (CASSCF-MRCI) level of theory (def2-SVP basis set [69]) in a three-dimensional search space (see the Supplemental Material [24] for details). The calculated potential energy surface (see Supplemental Material
Figs. S11 (a)-(c) [24]) shows a Pauli repulsion at $<4 \AA$ and an attractive interaction of $-0.1 \mathrm{eV}$ at longer distances. The strong interaction $\left(\sim 4 k_{B} T\right)$ is caused by dispersion forces of the large iodine atoms. We found that the fragment contact lifetime increased from 34 to 204 ps when using the force field parametrized against the results of our CASSCFMRCI calculations compared to the standard general Amber force field (Supplemental Material Fig. S12 [24]). We conclude that the dispersion force is sufficient to hold the radical pair in a mutual solvent pocket for a few hundred picoseconds. This is much longer than what was previously assumed in investigations using spectroscopy and $x$-ray scattering. [15,16,21,23,66,70,71].

The long contact lifetime means that the incomplete formation of the photoisomer cannot be explained by solvent escape [15,20-23]. Instead, a chemically inactive form of the geminate pair must exist. Although our data do not conclusively reveal the reason for this, we consider two possible explanations. First, photoisomer formation may be promoted by vibrational excitation of the $\mathrm{CH}_{2} \mathrm{I}^{\bullet}$ fragment. When the vibrational excitation is dissipated, the photoisomerization reaction proceeds at a much slower rate $\left(\tau_{2}\right)$ [72]. Alternatively, a competing reaction pathway, e.g., the formation of a solvent-solute complex $\left(\mathrm{I}^{\bullet} \cdots \mathrm{C}_{6} \mathrm{H}_{12}\right.$ [73-75]), hydrogen atom abstraction from the solvent, or loss of spin correlation, could generate a distribution of active and chemically inactive geminate pairs. The "active" species would form the photoisomer within the first phase $\left(\tau_{1}\right)$ and the "inhibited" species would form the photoisomer with the second phase $\left(\tau_{2}\right)$. We consider the formation of triplet radical pairs to be plausible, since the spin flip could occur in the excited state, prior to separation of the fragments.

Conclusion.-In conclusion, we have visualized the dissociation reaction of $\mathrm{CH}_{2} \mathrm{I}_{2}$ in solution using femtosecond time-resolved wide angle $\mathrm{x}$-ray scattering. The analysis yields a comprehensive movie for the first nanosecond of the reaction (Fig. 3). The iodine-carbon bond is broken directly after photoexcitation [(I) in Fig. 3], the $\mathrm{I}^{\bullet}$ and $\mathrm{CH}_{2} \mathrm{I}^{\circ}$ fragments fly apart until they collide with a

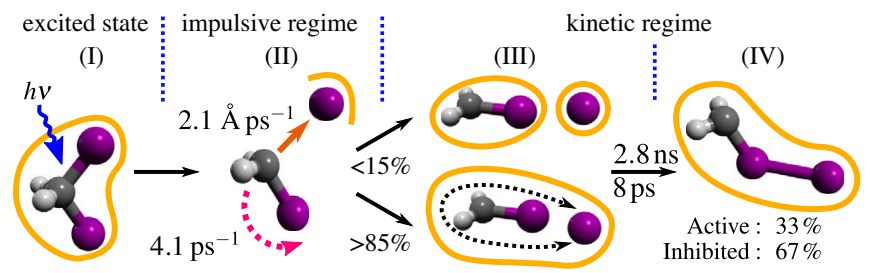

FIG. 3. Schematic representation of the revised mechanism of the photoisomer formation within $1 \mathrm{~ns}$. The excited state (I) leads to dissociation. In the impulsive regime (II), the rotational and translational speed of the fragments are given, and the time constants in the kinetic regime (III) and (IV) are lifetimes. The delineating orange lines represent the solvation shells of the structures. The black dotted line indicates the positional distribution of the I atom within the solvent shell. 
solvent molecule, and the $\mathrm{CH}_{2} \mathrm{I}^{\bullet}$ radical rotates [(II) in Fig. 3]. The translational motion of the fragments is stopped by the solvent collision, but the rotational motion is not fully inhibited. As a result, the majority of radical pairs stay within the solvent shell [(III) in Fig. 3]. We find that the radical pairs within the solvent cage are surprisingly stable, due to previously unrecognized dispersion forces between the heavy iodine atoms, but that a notable fraction of them is unreactive [(IV) in Fig. 3]. Accordingly, the photoisomer production proceeds biphasically on picoand nanosecond timescales. The sensitivity of timeresolved wide angle $\mathrm{x}$-ray scattering toward relative atomic positions, without the requirement of a covalent bond, was essential in uncovering this comprehensive, microscopic reaction mechanism in solution.

Use of the Linac Coherent Light Source (LCLS), SLAC National Accelerator Laboratory, is supported by the U.S. Department of Energy, Office of Science, Office of Basic Energy Sciences under Contract No. DE-AC0276SF00515. The experiments at SACLA were performed at BL3 with the approval of the Japan Synchrotron Radiation Research Institute (JASRI) (Proposal No. 2016A8037). S. W. acknowledges the European Research Council for support (Grants No. 6581802 and No. 279944). The DTU-affiliated authors gratefully acknowledge DANSCATT for support of the beam time activities. E. B. and S. W. further acknowledge support from Interreg. M. H., K. A., and J. S. H. were supported by the Deutsche Forschungsgemeinschaft (Grant No. HU 1971-1/1, No. HU 1971-3/1, and No. HU 1971-4/1).

*westenho@chem.gu.se

†Present address: PULSE Institute, SLAC National Laboratory, Menlo Park, California 94305, USA.

[1] J. Zheng, K. Kwak, J. Asbury, X. Chen, I. R. Piletic, and M. D. Fayer, Ultrafast dynamics of solute-solvent complexation observed at thermal equilibrium in real time, Science 309, 1338 (2005).

[2] P. Hamm and M. T. Zanni, Concepts and Methods of 2D Infrared Spectroscopy (Cambridge University Press, Cambridge, England, 2011).

[3] N. F. A. van der Vegt, K. Haldrup, S. Roke, J. Zheng, M. Lund, and H. J. Bakker, Water-mediated ion pairing: Occurrence and relevance, Chem. Rev. 116, 7626 (2016).

[4] M. P. Minitti, J. S. Robinson, R. N. Coffee, S. Edstrom, S. Gilevich, J. M. Glownia, E. Granados, P. Hering, M. C. Hoffmann, A. Miahnahri, D. Milathianaki, W. Polzin, D. Ratner, F. Tavella, S. Vetter, M. Welch, W. E. White, and A. R. Fry, Optical laser systems at the Linac Coherent Light Source, J. Synchrotron Radiat. 22, 526 (2015).

[5] T. Ishikawa, S. A. Hayes, S. Keskin, G. Corthey, M. Hada, K. Pichugin, A. Marx, J. Hirscht, K. Shionuma, K. Onda, Y. Okimoto, S. Y. Koshihara, T. Yamamoto, H. Cui, M. Nomura, Y. Oshima, M. Abdel-Jawad, R. Kato, and R. J. D. Miller, Direct observation of collective modes coupled to molecular orbital-driven charge transfer, Science 350, 1501 (2015).

[6] S. E. Canton et al., Visualizing the non-equilibrium dynamics of photoinduced intramolecular electron transfer with femtosecond X-ray pulses, Nat. Commun. 6, 6359 (2015).

[7] K. H. Kim, J. G. Kim, S. Nozawa, T. Sato, K. Y. Oang, T. W. Kim, H. Ki, J. Jo, S. Park, C. Song, T. Sato, K. Ogawa, T. Togashi, K. Tono, M. Yabashi, T. Ishikawa, J. Kim, R. Ryoo, J. Kim, H. Ihee, and S.-i. Adachi, Direct observation of bond formation in solution with femtosecond X-ray scattering, Nature (London) 518, 385 (2015).

[8] E. Biasin et al., Femtosecond X-Ray Scattering Study of Ultrafast Photoinduced Structural Dynamics in Solvated $\left.[\text { Co(terpy })_{2}\right]^{2+}$, Phys. Rev. Lett. 117, 013002 (2016).

[9] B. Wolter, M. G. Pullen, A. T. Le, M. Baudisch, K. Doblhoff-Dier, A. Senftleben, M. Hemmer, C. D. Schröter, J. Ullrich, T. Pfeifer, R. Moshammer, S. Gräfe, O. Vendrell, C. D. Lin, and J. Biegert, Ultrafast electron diffraction imaging of bond breaking in di-ionized acetylene, Science 354, 308 (2016).

[10] A. R. Attar, A. Bhattacherjee, C. D. Pemmaraju, K. Schnorr, K. D. Closser, D. Prendergast, and S. R. Leone, Femtosecond $\mathrm{x}$-ray spectroscopy of an electrocyclic ring-opening reaction, Science 356, 54 (2017).

[11] Y. Liu, S. L. Horton, J. Yang, J. P. F. Nunes, X. Shen, T. J. A. Wolf, R. Forbes, C. Cheng, B. Moore, M. Centurion, K. Hegazy, R. Li, M.-F. Lin, A. Stolow, P. Hockett, T. Rozgonyi, P. Marquetand, X. Wang, and T. Weinacht, Spectroscopic and Structural Probing of Excited-State Molecular Dynamics with Time-Resolved Photoelectron Spectroscopy and Ultrafast Electron Diffraction, Phys. Rev. X 10, 021016 (2020).

[12] N. Li, T. P. Keane, S. S. Veroneau, R. G. Hadt, D. Hayes, L. X. Chen, and D. G. Nocera, Template-stabilized oxidic nickel oxygen evolution catalysts, Proc. Natl. Acad. Sci. U.S.A. 117, 16187 (2020).

[13] D. Kinschel et al., Femtosecond X-ray emission study of the spin cross-over dynamics in haem proteins, Nat. Commun. 11, 4145 (2020).

[14] P. M. Kroger, P. C. Demou, and S. J. Riley, Polyhalide photofragment spectra. I. Two-photon two-step photodissociation of methylene iodide, J. Chem. Phys. 65, 1823 (1976).

[15] A. N. Tarnovsky, V. Sundström, E. Åkesson, and T. Pascher, Photochemistry of diiodomethane in solution studied by femtosecond and nanosecond laser photolysis. Formation and dark reactions of the $-\mathrm{CH}_{2} \mathrm{I}-\mathrm{I}$ isomer photoproduct and its role in cyclopropanation of olefins, J. Phys. Chem. A 108, 237 (2004).

[16] J. Davidsson, J. Poulsen, M. Cammarata, P. Georgiou, R. Wouts, G. Katona, F. Jacobson, A. Plech, M. Wulff, G. Nyman, and R. Neutze, Structural Determination of a Transient Isomer of $\mathrm{CH}_{2} \mathrm{I}_{2}$ by Picosecond X-Ray Diffraction, Phys. Rev. Lett. 94, 245503 (2005).

[17] X. Zheng and D. L. Phillips, Solvation can open the photoisomerization pathway for the direct photodissociation reaction of diiodomethane: Transient resonance Raman observation of the isodiiodomethane photoproduct from ultraviolet excitation of diiodomethane in the solution phase, J. Phys. Chem. A 104, 6880 (2000). 
[18] D. Phillips, W. Fang, and X. Zheng, Isodiiodomethane is the methylene transfer agent in cyclopropanation reactions with olefins using ultraviolet photolysis of diiodomethane in solutions: A density functional theory investigation of the reactions of isodiiodomethane, iodomethyl radical, and iodomethyl cation with ethylene, J. Am. Chem. Soc. 123, 4197 (2001).

[19] V. A. Borin, S. M. Matveev, D. S. Budkina, P. Z. El-Khoury, and A. N. Tarnovsky, Direct photoisomerization of $\mathrm{CH}_{2} \mathrm{I}_{2}$ vs. $\mathrm{CHBr}_{3}$ in the gas phase: a joint 50 fs experimental and multireference resonance-theoretical study, Phys. Chem. Chem. Phys. 18, 28883 (2016).

[20] B. J. Schwartz, J. C. King, J. Z. Zhang, and C. B. Harris, Direct femtosecond measurements of single collision dominated geminate recombination times of small molecules in liquids, Chem. Phys. Lett. 203, 503 (1993).

[21] J. Franck and E. Rabinowitsch, Some remarks about free radicals and the photochemistry of solutions, Trans. Faraday Soc. 30, 120 (1934).

[22] T. Kühne and P. Vöhringer, Vibrational relaxation and geminate recombination in the femtosecond-photodissociation of triiodide in solution, J. Chem. Phys. 105, 10788 (1996).

[23] U. Banin and S. Ruhman, Ultrafast photodissociation of $\mathrm{I}_{3}$. Coherent photochemistry in solution, J. Chem. Phys. 98, 4391 (1993).

[24] See Supplemental Material at http://link.aps.org/ supplemental/10.1103/PhysRevLett.125.226001 for a detailed description of the TRWAXS experiments, molecular dynamics simulations, quantum mechanics calculations, signal processing, data analysis, transient absorption data, and additional TRWAXS data, which includes Refs. [25-59].

[25] K. S. Kjaer, T. B. van Driel, J. Kehres, K. Haldrup, D. Khakhulin, K. Bechgaard, M. Cammarata, M. Wulff, T. J. Sorensen, and M. M. Nielsen, Introducing a standard method for experimental determination of the solvent response in laser pump, x-ray probe time-resolved wide-angle $\mathrm{x}$-ray scattering experiments on systems in solution, Phys. Chem. Chem. Phys. 15, 15003 (2013).

[26] M. Chollet, R. Alonso-Mori, M. Cammarata, D. Damiani, J. Defever, J. T. Delor, Y. Feng, J. M. Glownia, J. B. Langton, S. Nelson, K. Ramsey, A. Robert, M. Sikorski, S. Song, D. Stefanescu, V. Srinivasan, D. Zhu, H. T. Lemke, and D. M. Fritz, The x-ray pump-probe instrument at the Linac coherent light source, J. Synchrotron Radiat. 22, 503 (2015).

[27] K. Tono, Y. Inubushi, T. Sato, T. Togashi, H. Ohashi, H. Kimura, S. Takahashi, K. Takeshita, H. Tomizawa, S. Goto, and M. Yabashi, Beamline for x-ray free electron laser of SACLA, J. Phys. Conf. Ser. 425, 072006 (2013).

[28] K. Tono, T. Togashi, Y. Inubushi, T. Sato, T. Katayama, K. Ogawa, H. Ohashi, H. Kimura, S. Takahashi, K. Takeshita, H. Tomizawa, S. Goto, T. Ishikawa, and M. Yabashi, Beamline, experimental stations and photon beam diagnostics for the hard x-ray free electron laser of SACLA, New J. Phys. 15, 083035 (2013).

[29] T. Sato, T. Togashi, K. Ogawa, T. Katayama, Y. Inubushi, K. Tono, and M. Yabashi, Highly efficient arrival timing diagnostics for femtosecond x-ray and optical laser pulses, Appl. Phys. Express 8, 012702 (2015).
[30] T. Katayama, S. Owada, T. Togashi, K. Ogawa, P. Karvinen, I. Vartiainen, A. Eronen, C. David, T. Sato, K. Nakajima, Y. Joti, H. Yumoto, H. Ohashi, and M. Yabashi, A beam branching method for timing and spectral characterization of hard x-ray free-electron lasers, Struct. Dyn. 3, 034301 (2016).

[31] T. Sato, T. Togashi, K. Tono, Y. Inubushi, H. Tomizawa, Y. Tanaka, S. Adachi, K. Nakamura, R. Kodama, and M. Yabashi, Development of ultrafast pump and probe experimental system at SACLA, J. Phys. Conf. Ser. 425, 092009 (2013).

[32] K.-I. Saitow, Y. Naitoh, K. Tominaga, and K. Yoshihara, Photo-induced reactions of $\mathrm{CH}_{2} \mathrm{I}_{2}$ in solution studied by the ultrafast transient absorption spectroscopy, Chem. Phys. Lett. 262, 621 (1996).

[33] Y.-L. Li, D. Wang, and D. L. Phillips, Time-resolved resonance Raman spectroscopy and density functional theory investigation of the $\mathrm{CH}_{2} \mathrm{I}-\mathrm{I}$ isomer and $\mathrm{CH}_{2} \mathrm{I}_{2} \cdots \mathrm{I}$ molecular complex products produced from ultraviolet photolysis of $\mathrm{CH}_{2} \mathrm{I}_{2}$ in the solution phase: Comparison of the structure and chemical reactivity of polyhalomethane isomers and polyhalomethane-halogen atom molecular complexes, J. Chem. Phys. 117, 79317941 (2002).

[34] A. N. Tarnovsky, M. Wall, M. Gustafsson, N. Lascoux, V. Sundström, and E. A kesson, Ultrafast study of the photodissociation of bromoiodomethane in acetonitrile upon 266 nm excitation, J. Phys. Chem. A 106, 5999 (2002).

[35] E. Jones, T. Oliphant, P. Peterson et al., SciPy: Open source scientific tools for Python (2001-), [Online; accessed].

[36] M. Newville, T. Stensitzki, D. B. Allen, and A. Ingargiola, LMFIT: Non-linear least-square minimization and curvefitting for python, 2014.

[37] K. Haldrup, M. Christensen, and M. M. Nielsen, Analysis of time-resolved $\mathrm{x}$-ray scattering data from solution-state systems, Acta Crystallogr. Sect. A 66, 261 (2010).

[38] T. B. van Driel, K. S. Kjaer, E. Biasin, K. Haldrup, H. T. Lemke, and M. M. Nielsen, Disentangling detector data in XFEL studies of temporally resolved solution state chemistry, Faraday Discuss. 177, 443 (2015).

[39] T. K. Kim, J. H. Lee, M. Wulff, Q. Kong, and H. Ihee, Spatiotemporal kinetics in solution studied by time-resolved x-ray liquidography (solution scattering), Chem. Phys. Chem. 10, 1958 (2009).

[40] A. O. Dohn, E. Biasin, K. Haldrup, M. M. Nielsen, N. E. Henriksen, and K. B. M. ller, On the calculation of x-ray scattering signals from pairwise radial distribution functions, J. Phys. B 48, 244010 (2015).

[41] R. D. B. Fraser, T. P. MacRae, and E. Suzuki, An improved method for calculating the contribution of solvent to the X-ray diffraction pattern of biological molecules, J. Appl. Crystallogr. 11, 693 (1978).

[42] J. Wang, R. M. Wolf, J. W. Caldwell, P. A. Kollman, and D. A. Case, Development and testing of a general amber force field., J. Comput. Chem. 25, 1157 (2004).

[43] D. van der Spoel, P. J. van Maaren, and C. Caleman, GROMACS molecule and liquid database, Bioinformatics 28, 752 (2012).

[44] S. Pronk, S. Páll, R. Schulz, P. Larsson, P. Bjelkmar, R. Apostolov, M. R. Shirts, J. C. Smith, P. M. Kasson, D. van der Spoel, B. Hess, and E. Lindahl, GROMACS4.5: A high- 
throughput and highly parallel open source molecular simulation toolkit, Bioinformatics 29, 845 (2013).

[45] W. F. van Gunsteren and H. J. C. Berendsen, A leap-frog algorithm for stochastic dynamics, Mol. Simul. 1, 173 (1988).

[46] H. J. C. Berendsen, J. P. M. Postma, A. DiNola, and J. R. Haak, Molecular dynamics with coupling to an external bath, J. Chem. Phys. 81, 3684 (1984).

[47] B. Hess, P-LINCS: A parallel linear constraint solver for molecular simulation, J. Chem. Theory Comput. 4, 116 (2008).

[48] F. Neese, The ORCA program system, Wiley Interdisciplinary Rev. 2, 73 (2012).

[49] F. Neese, Software update: The ORCA program system, version 4.0, Wiley Interdisciplinary Rev. 2, e1327 (2017).

[50] K. A. Peterson, D. Figgen, M. Dolg, and H. Stoll, Energyconsistent relativistic pseudopotentials and correlation consistent basis sets for the $4 \mathrm{~d}$ elements YPd, J. Chem. Phys. 126, 124101 (2007).

[51] M. J. Abraham, T. Murtola, R. Schulz, S. Páll, J. C. Smith, B. Hess, and E. Lindahl, GROMACs: High performance molecular simulations through multi-level parallelism from laptops to supercomputers, SoftwareX 1-2, 19 (2015).

[52] H. J. C. Berendsen, J. P. M. Postma, W. F. van Gunsteren, A. DiNola, and J. R. Haak, Molecular-dynamics with coupling to an external bath, J. Chem. Phys. 81, 3684 (1984).

[53] D. Lavalette, C. Tétreau, M. Tourbez, and Y. Blouquit, Microscopic viscosity and rotational diffusion of proteins in a macromolecular environment, Biophys. J. 76, 2744 (1999).

[54] M. Horng, J. Gardecki, and M. Maroncelli, Rotational dynamics of coumarin 153: Time-dependent friction, dielectric friction, and other nonhydrodynamic effects, J. Phys. Chem. A 101, 1030 (1997).

[55] T. G. Devi and K. Kumar, Raman bandshape analysis of o-chlorobenzaldehyde: Microviscosity-dependent study, J. Raman Spectrosc. 35, 835 (2004).

[56] A. Gierer and K. Wirtz, Molekulare theorie der mikroreibung, Z. Naturforsch. 8A, 532 (1953).

[57] N. Michaud-Agrawal, E. J. Denning, T. B. Woolf, and O. Beckstein, MDANALYSIS: A toolkit for the analysis of molecular dynamics simulations, J. Comput. Chem. 32, 2319 (2011).

[58] R. J. Gowers, M. Linke, J. Barnoud, T. J. E. Reddy, M. N. Melo, S. L. Seyler, J. Domański, D. L. Dotson, S. Buchoux, I. M. Kenney, and O. Beckstein, MDANALYSIS: A python package for the rapid analysis of molecular dynamics simulations, in Proceedings of the 15th Python in Science Conference, edited by S. Benthall and S. Rostrup (2016), pp. 98-105.

[59] M. D. Hanwell, D. E. Curtis, D. C. Lonie, T. Vandermeersch, E. Zurek, and G. R. Hutchison, AVOGADRO: An advanced semantic chemical editor, visualization, and analysis platform, J. Cheminform. 4 (2012).

[60] M. Harmand, R. Coffee, M. Bionta, M. Chollet, D. French, D. Zhu, D. Fritz, H. Lemke, N. Medvedev, B. Ziaja, S. Toleikis, and M. Cammarata, Achieving few-femtosecond time-sorting at hard X-ray free-electron lasers, Nat. Photonics 7, 215 (2013).

[61] E. Biasin, T. B. van Driel, G. Levi, M. G. Laursen, A. O. Dohn, A. Moltke, P. Vester, F. B. K. Hansen, K. S. Kjaer, T.
Harlang, R. Hartsock, M. Christensen, K. J. Gaffney, N. E. Henriksen, K. B. Møller, K. Haldrup, and M. M. Nielsen, Anisotropy enhanced X-ray scattering from solvated transition metal complexes, J. Synchrotron Radiat. 25, 306 (2018).

[62] J. G. Kim et al., Mapping the emergence of molecular vibrations mediating bond formation, Nature (London) $\mathbf{5 8 2}$, 520 (2020).

[63] A. Plech, M. Wulff, S. Bratos, F. Mirloup, R. Vuilleumier, F. Schotte, and P. A. Anfinrud, Visualizing Chemical Reactions in Solution by Picosecond X-Ray Diffraction, Phys. Rev. Lett. 92, 125505 (2004).

[64] J. Vincent, M. Andersson, M. Eklund, A. B. Wöhri, M. Odelius, E. Malmerberg, Q. Kong, M. Wulff, R. Neutze, and J. Davidsson, Solvent dependent structural perturbations of chemical reaction intermediates visualized by time-resolved X-ray diffraction, J. Chem. Phys. 130, 154502 (2009).

[65] S. Park, J. Choi, H. Ki, K. H. Kim, K. Y. Oang, H. Roh, J. Kim, S. Nozawa, T. Sato, S.-i. Adachi, J. Kim, and H. Ihee, Fate of transient isomer of $\mathrm{CH}_{2} \mathrm{I}_{2}$ : Mechanism and origin of ionic photoproducts formation unveiled by time-resolved X-ray liquidography, J. Chem. Phys. 150, 224201 (2019).

[66] H. Ihee, M. Lorenc, T. K. Kim, Q. Y. Kong, M. Cammarata, J. H. Lee, S. Bratos, and M. Wulff, Ultrafast X-ray diffraction of transient molecular structures in solution, Science 309, 1223 (2005).

[67] K.-W. Jung, T. Ahmadi, and M. El-Sayed, Bull. Korean Chem. Soc. 18, 1274 (1997).

[68] Because of technical implementation at the XFEL, the TRWAXS data was recorded in two time ranges. Less repeats were recorded for the time range containing the time points $t \geq 10 \mathrm{ps}$.

[69] F. Weigend and R. Ahlrichs, Balanced basis sets of split valence, triple zeta valence and quadruple zeta valence quality for $\mathrm{H}$ to $\mathrm{Rn}$ : Design and assessment of accuracy, Phys. Chem. Chem. Phys. 7, 3297 (2005).

[70] Q. Liu, J. K. Wang, and A. H. Zewail, Femtosecond dynamics of dissociation and recombination in solvent cages, Nature (London) 364, 427 (1993).

[71] H. Ihee, Visualizing solution-phase reaction dynamics with time-resolved x-ray liquidography, Acc. Chem. Res. 42, 356 (2009).

[72] S. Schott, L. Ress, J. Hrušák, P. Nuernberger, and T. Brixner, Identification of photofragmentation patterns in trihalide anions by global analysis of vibrational wavepacket dynamics in broadband transient absorption data, Phys. Chem. Chem. Phys. 18, 33287 (2016).

[73] S. R. Logan, R. Bonneau, J. Joussot-Dubien, and P. F. de Violet, Solvent-iodine atom charge transfer absorption in liquid alkanes and cycloalkanes, J. Chem. Soc., Faraday Trans. 1 71, 2148 (1975).

[74] A. L. Harris, M. Berg, and C. B. Harris, Studies of chemical reactivity in the condensed phase. I. The dynamics of iodine photodissociation and recombination on a picosecond time scale and comparison to theories for chemical reactions in solution, J. Chem. Phys. 84, 788 (1986).

[75] C. P. Anderson, K. G. Spears, K. R. Wilson, and R. J. Sension, Solvent dependent branching between C-I and $\mathrm{C}-\mathrm{Br}$ bond cleavage following $266 \mathrm{~nm}$ excitation of $\mathrm{CH}_{2}$ BrI, J. Chem. Phys. 139, 194307 (2013). 